\title{
Search for invisible or lepton-flavor-violating Upsilon decays at BABAR
}

\author{
Justin ALBERT*, representing the BABAR Collaboration \\ Dept. of Physics \& Astronomy, Univ. of Victoria, 3800 Finnerty Rd., Victoria, B.C. V8P 5 C2 \\ Canada \\ E-mail: jalbert@uvic.ca
}

Both invisible and lepton-flavor-violating Upsilon decays are unobservably rare in the Standard Model, but are each predicted to occur in several beyond-Standard-Model scenarios. We search for invisible, as well as for lepton-flavor-violating, Upsilon decays in a sample of $117 \times 10^{6}$ $\Upsilon(3 S)$ decays collected with the BABAR detector. To search for invisible Upsilon decays, we select events containing the decay $\Upsilon(3 S) \rightarrow \pi^{+} \pi^{-} \Upsilon(1 S)$ and search for evidence of an undetectable $\Upsilon(1 S)$ decay recoiling against the dipion system. We set upper limits on the invisible branching fraction $\mathscr{B}(\Upsilon(1 S) \rightarrow$ invisible $)<3.0 \times 10^{-4}$, and on the lepton-flavor-violating branching fractions $\mathscr{B}\left(\Upsilon(3 S) \rightarrow e^{ \pm} \tau^{\mp}\right)<5.0 \times 10^{-6}$ and $\mathscr{B}\left(\Upsilon(3 S) \rightarrow \mu^{ \pm} \tau^{\mp}\right)<4.1 \times 10^{-6}$, all at the $90 \%$ confidence level.

European Physical Society Europhysics Conference on High Energy Physics, EPS-HEP 2009,

July 16-22 2009

Krakow, Poland

\footnotetext{
${ }^{*}$ Speaker.
} 


\section{Introduction}

In the Standard Model (SM), invisible decays of $\Upsilon$ mesons can proceed via $b \bar{b}$ annihilation into a $v \bar{v}$ pair with a branching fraction $\mathscr{B}(\Upsilon \rightarrow$ invisible $) \approx 1 \times 10^{-5}$ [1]. Charged leptonflavor violating (LFV) decays in the SM, including those of the $r$, are suppressed by the ratio $\left(\Delta m_{v}^{2} / M_{W}^{2}\right)^{2} \lesssim 10^{-48}[2,3,4]$. This implies that both types of processes are presently unobservably rare in the SM. However, models containing low-mass dark matter candidates can enhance the invisible branching fraction of the $\Upsilon$ to the level of $10^{-4}$ to $10^{-3}$ [5], and a wide range of supersymmetric and other models with new physics in the TeV range can enhance LFV branching fractions of the $r$ to present experimental limits $[6,7,8,9]$. We thus search for both types of processes in a sample of approximately 100 million $\Upsilon(3 S)$ decays collected at BABAR. The BABAR detector at the PEP-II storage ring is described in detail elsewhere [10], and all signal processes and generic $\Upsilon(3 S)$ decays are modeled using a Monte Carlo (MC) simulation based on GEANT 4 [11].

\section{Search for invisible $\Upsilon(1 S)$ decays}

We search for $\Upsilon(3 S) \rightarrow \pi^{+} \pi^{-} \Upsilon(1 S)$ events, which are generated in MC according to the matrix elements measured by the CLEO collaboration [12]. In signal events, the mass recoiling against the dipion $\left(M_{\mathrm{rec}}\right)$ peaks at the $\Upsilon(1 S)$ mass $\left(9.4603 \mathrm{GeV} / c^{2}\right.$ [13]). The same is true for background events in which a real $\Upsilon(3 S) \rightarrow \pi^{+} \pi^{-} \Upsilon(1 S)$ transition occurs but the $\Upsilon(1 S)$ final-state particles are undetected ("peaking background"). However, the dominant background containing a pair of low-momentum pions does not exhibit this structure ("non-peaking background"). The analysis strategy is as follows: first apply selection criteria to suppress background, primarily the non-peaking component; then fit the resulting $M_{\text {rec }}$ spectrum to measure the peaking component (signal plus peaking background).

We select events by requiring that there are exactly two tracks originating from the interaction point (IP), with opposite electric charge. The dipion system is required to have an invariant mass satisfying $M_{\pi \pi} \in[0.25,0.95] \mathrm{GeV} / c^{2}$, compatible with kinematic boundaries after allowing for resolution effects. The dipion recoil mass is $M_{\text {rec }}^{2}=s+M_{\pi \pi}^{2}-2 \sqrt{s} E_{\pi \pi}^{*}$, where $E_{\pi \pi}^{*}$ is the center-of-mass (CM) energy of the dipion system and $\sqrt{s}=10.3552 \mathrm{GeV} / c^{2}$. We require that $M_{\text {rec }} \in[9.41,9.52] \mathrm{GeV} / c^{2}$. The efficiency of this selection for signal events is about $64 \%$.

We reject most of this remaining background with a multivariate analysis (MVA), implemented as a random forest of decision trees [14]. We use the following variables as inputs to the MVA: the probability that the pions originate from a common vertex; the laboratory polar angle and transverse momentum of the dipion system; the total number of charged tracks, IP tracks or otherwise, reconstructed in the event; booleans that indicate whether either pion has passed electron, kaon, or muon identification criteria; the cosine of the angle (in the CM frame) between the highest-energy photon $\left(\gamma_{1}\right)$ and the normal to the decay plane of the dipion system; the energy in the laboratory frame of the $\gamma_{1}$; the total neutral energy in the CM frame; and the multiplicity of $K_{L}^{0}$ candidates, defined using the shape and magnitude of the shower resulting from interactions in the calorimeter. The total efficiency of all trigger and event selection requirements is determined from signal-MC simulation to be $16.4 \%$. 
We extract the peaking yield by an extended unbinned maximum likelihood fit, with the nonpeaking background described by a first-order polynomial. The signal and the peaking background should have the same shape in $M_{\mathrm{rec}}$. We describe this shape using a modified Gaussian function with a common peak position, independent left and right widths, and non-Gaussian tails.

The fit then determines all of the parameters of the non-peaking background probability density function (PDF), the yield of the non-peaking background, and the yield of the peaking component. The fit is shown in Fig. 1. The result for the peaking yield is $2326 \pm 105$ events. The systematic uncertainty on that yield associated with the fixed parameters in the signal PDF is estimated by varying those parameters in the fit. We find an uncertainty of 18 events.

We next estimate the contribution of background to the peak. The MC simulation predicts $1019 \Upsilon(1 S) \rightarrow e^{+} e^{-}$events, $1007 \Upsilon(1 S) \rightarrow \mu^{+} \mu^{-}$events, $92 \Upsilon(1 S) \rightarrow \tau^{+} \tau^{-}$events, and $2.9 \pm 1.3$ $\Upsilon(1 S) \rightarrow$ hadrons events. These predictions depend upon branching fractions which have significant uncertainties [13] and on the accuracy of the modeling of event reconstruction and selection. We use additional data and MC subsamples to test and correct the MC prediction of 2122 total events, resulting in a corrected estimate of $(2451 \pm 38)$ peaking background events.

A number of multiplicative systematic corrections and uncertainties to the peaking background also enter when the extracted signal yield is converted to the $\Upsilon(1 S) \rightarrow$ invisible branching fraction. We find the total systematic uncertainty in common between signal and peaking background to be $4.8 \%$. The final corrected prediction for the peaking background is thus ( $2444 \pm 123)$ events. Fitting the data, we then obtain a signal yield of $(-118 \pm 105$ (stat) \pm 124 (syst) ) events. Using a determination of the signal efficiency and a count of the total number of $\Upsilon(3 S)$ events in the data sample, we determine that $\mathscr{B}(\Upsilon(1 S) \rightarrow$ invisible $)=(-1.6 \pm 1.4($ stat $) \pm 1.6($ syst $)) \times 10^{-4}$. Lacking evidence for this decay, we use a Bayesian technique to set an upper limit on the branching fraction of $\mathscr{B}(\Upsilon(1 S) \rightarrow$ invisible $)<3.0 \times 10^{-4}$ at $90 \%$ confidence level (CL) [15].

\section{Search for lepton-flavor-violating $\Upsilon(3 S)$ decays}

The signature of the signal $\Upsilon(3 S) \rightarrow \ell^{ \pm} \tau^{\mp}$ events consists of two oppositely-charged tracks consistent with originating from the primary IP, with opening angle greater than $90^{\circ}$ in the $\mathrm{CM}$ frame: a primary electron or muon with CM momentum close to the beam energy $E_{B}=\sqrt{s} / 2$, and a secondary charged lepton or charged pion from the $\tau$ decay. If the $\tau$ decays leptonically, we require that the primary and $\tau$-daughter leptons are of different flavor. If the $\tau$ decays hadronically, we require one or two additional neutral pions from this decay. Thus we define four signal channels, consisting of leptonic and hadronic $\tau$ decay modes for the $\Upsilon(3 S) \rightarrow e^{ \pm} \tau^{\mp}$ and $\Upsilon(3 S) \rightarrow \mu^{ \pm} \tau^{\mp}$ searches, referred to as the leptonic and hadronic $e \tau$ and $\mu \tau$ channels. The main source of background comes from $\tau$-pair production, which constitutes an irreducible background decaying to the same final state as the signal process. There is also a reducible background contribution to the $e \tau$ channels from Bhabha events in which one of the electrons is misidentified, and to the $\mu \tau$ channels from $\mu$-pair events in which one of the muons is misidentified or decays in flight, or an electron is generated in a material interaction.

We require $\cos \left(\theta_{\text {miss }}^{\text {lab }}\right)<0.9, \cos \left(\theta_{\text {miss }}^{C M}\right)>-0.9, M_{V I S} / \sqrt{s}<0.95$, and $\left(\vec{p}_{1}+\vec{p}_{2}\right)_{\perp} /\left(\sqrt{s}-\left|\vec{p}_{1}\right|-\right.$ $\left.\left|\vec{p}_{2}\right|\right)>0.2$. Here $\theta_{\text {miss }}^{\text {lab }}\left(\theta_{\text {miss }}^{C M}\right)$ is the polar angle of the missing momentum in the lab (CM) frame, $M_{V I S}$ is the mass of the 4-vector obtained by summing the 4-vectors of the two tracks and those of 

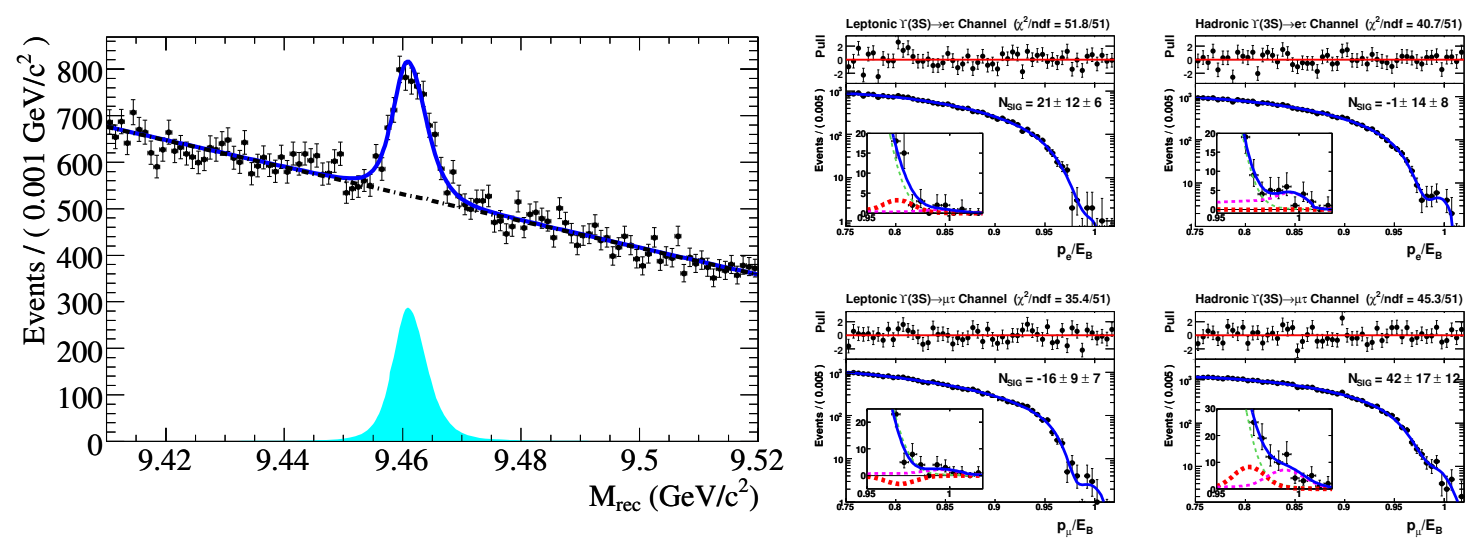

Figure 1: (Left) The maximum likelihood fit to the dipion recoil mass for data in the invisible subsample. The components of the fit are the non-peaking background (dash-dotted line) and the peaking component (solid filled). The total fit function is also shown (solid line). (Right) Fit results for the four LFV signal channels. The dashed lines indicate three component PDFs, the solid line indicates their sum. The thin green dashed line is the $\tau$-pair background PDF, the medium magenta dashed line is the Bhahba background PDF for the $e \tau$ channels and the $\mu$-pair background PDF for the $\mu \tau$ channels, and the thick red dashed line is the signal PDF. The insets show a close-up of the region $0.95<x<1.02 . N_{S I G}$ is the signal yield extracted by the fit.

any additional photons in the event, and $\vec{p}_{1,2}$ are the CM momenta of the two tracks. These requirements suppress Bhabha and $\mu$-pair events, two-photon processes, and beam-gas interactions.

Particle identification is performed by combining information from all detector subsystems using a multivariate analysis [16]. The CM momentum of the primary lepton, defined as the $e(\mu)$ for the $e \tau(\mu \tau)$ channels, is required to satisfy $p / E_{B}>0.75$. Additional kinematic selection criteria are then applied to further suppress the Bhabha and $\mu$-pair backgrounds. For the hadronic $\tau$-decay channels, the CM momentum of the $\tau$-daughter charged pion is required to satisfy $p / E_{B}<0.8$. In addition, the masses of the $\pi^{ \pm} \pi^{0}$ and $\pi^{ \pm} \pi^{0} \pi^{0}$ systems (for events containing two neutral pions only) are required to be consistent with the masses of the $\rho$ and $a_{1}$ mesons, $m_{\rho}=0.77 \mathrm{GeV} / c^{2}$ and $m_{a_{1}}=1.26 \mathrm{GeV} / c^{2}$, respectively. For the leptonic $e \tau$ channel, the $\tau$-daughter muon is required to penetrate at least four layers of the muon detector. For the leptonic $\mu \tau$ channel, the tracks are required to not be back-to-back by requiring that the difference between the track azimuthal angles in the $\mathrm{CM}$ frame satisfies $\Delta \phi^{\mathrm{CM}}<172^{\circ}$. After including all selection requirements, typical signal efficiencies determined from MC are 4-6\%, including the $\tau$ decay branching fractions.

An unbinned, extended maximum likelihood fit is then performed using the discriminant variable $x=p_{1} / E_{B}$, the primary lepton $\mathrm{CM}$ momentum normalized to the beam-energy. The signal peaks at $x \approx 0.97$, while the $\tau$-pair background $x$ distribution is smooth and approaches zero as $x \rightarrow x_{M A X}$, where $x_{M A X} \approx 0.97$ is the effective kinematic endpoint for the lepton momentum in the decay $\tau^{-} \rightarrow \ell^{-} \bar{v}_{\ell} \nu_{\tau}$, boosted into the $\Upsilon(3 S)$ rest-frame. The $x$ distributions of the Bhabha/ $\mu$ pair backgrounds have a peaking component near $x=1, \sim 2.5-3 \sigma_{x}$ above the signal peak, where $\sigma_{x} \approx 0.01$ denotes the detector momentum resolution. We obtain signal yields of $(21 \pm 12 \pm 6)$, $(-1 \pm 14 \pm 8),(-16 \pm 9 \pm 7)$, and $(42 \pm 17 \pm 12)$ events in the leptonic $e \tau$, hadronic $e \tau$, leptonic $\mu \tau$, and hadronic $\mu \tau$ channels respectively, where the first uncertainties are statistical and the second 
systematic. The fit results are displayed in Fig. 1.

The signal yields are all consistent with zero. The upper limits are determined using a Bayesian extraction in which the prior is taken to be the step function $\theta(0)$. We determine that $B F(\Upsilon(3 S) \rightarrow$ $\left.e^{ \pm} \tau^{\mp}\right)<5.0 \times 10^{-6}$ and $B F\left(\Upsilon(3 S) \rightarrow \mu^{ \pm} \tau^{\mp}\right)<4.1 \times 10^{-6}$, both at 90\% CL [17].

\section{Conclusion}

We search for events in the processes $\Upsilon(1 S) \rightarrow$ invisible, $\Upsilon(3 S) \rightarrow e^{ \pm} \tau^{\mp}$, and $\Upsilon(3 S) \rightarrow \mu^{ \pm} \tau^{\mp}$. No significant signal is seen and we set upper limits at $90 \% \mathrm{CL}$ on each process. The results represent the first upper limit on $B F\left(\Upsilon(3 S) \rightarrow e^{ \pm} \tau^{\mp}\right)$, and a sensitivity improvement of factors of 4.9 and 8.3, respectively, on previous upper limits on $B F\left(\Upsilon(3 S) \rightarrow \mu^{ \pm} \tau^{\mp}\right)[18]$ and $B F(\Upsilon(1 S) \rightarrow$ invisible) $[19,20]$. The upper limit on the invisible decay process constrains specific models of dark matter [5], and the limits on the LFV processes constrain mass scales of new LFV contributions to $\Upsilon(3 S)$ decays.

\section{References}

[1] L.N. Chang, O. Lebedev, and J.N. Ng, Phys. Lett. B441, 419 (1998).

[2] G. Feinberg, Phys.Rev. 110, 1482 (1958).

[3] S. M. Bilenky and B. Pontecorvo, Phys. Lett. B61, 248 (1976).

[4] A. Strumia and F. Vissani, arXiv:hep-ph/0606054, (2007).

[5] R. McElrath, Phys. Rev. D 72, 103508 (2005).

[6] J. R. Ellis et al., Eur. Phys. J. C14, 319 (2000).

[7] J. R. Ellis, M. Raidal, and T. Yanagida, Phys. Lett. B581, 9 (2004).

[8] J.C. Pati and A. Salam, Phys. Rev. D10, 275 (1974).

[9] H. Georgi and S.L. Glashow, Phys. Rev. Lett. 32, 438 (1974).

[10] B. Aubert et al. (BABAR Collaboration), Nucl. Instrum. Methods Phys. Res., Sect. A 479, 1 (2002).

[11] S. Agostinelli et al. (GEANT4 Collaboration), Nucl. Instrum. Methods Phys. Res., Sect. A 506250 (2003).

[12] D. Cronin-Hennessy et al., Phys. Rev. D 76, 072001 (2007).

[13] C. Amsler et al. (Particle Data Group), Phys. Lett. B 667, 1 (2008).

[14] L. Breiman, Machine Learning 45, 5 (2001).

[15] B. Aubert et al. (BABAR Collaboration), arXiv:0908.2840v2 [hep-ex] (2009), submitted to Phys. Rev. Lett.

[16] I. Narsky, arXiv:physics/0507143v1 [physics.data-an] (2008).

[17] B. Aubert et al. (BABAR Collaboration), arXiv:0812.1021v2 [hep-ex] (2008).

[18] W. Love et al. (CLEO Collaboration), Phys. Rev. Lett. 101, 201601 (2008).

[19] P. Rubin et al. (CLEO Collaboration), Phys. Rev. D 75, 031104 (2007).

[20] O. Tajima et al. (BELLE Collaboration), Phys. Rev. Lett. 98, 132001 (2007). 